\title{
Thermal sensitivity is not changed by acute pain or afferent stimulation
}

\author{
ANDERS EKBLOM, PER HANSSON \\ From the Department of Physiology II, Karolinska Institutet, Stockholm, Sweden
}

SUMMARY The effect of conditioning stimulation on thermal sensitivity and clinical pain was studied in $\mathbf{4 0}$ patients and six healthy subjects. Thresholds regarding cold, warm and heat pain perception did not differ significantly between the painful and non-painful skin areas in patients or between patients and healthy subjects before stimulation. The patients received either $100 \mathrm{~Hz}$ TENS, $2 \mathrm{~Hz}$ TENS, $100 \mathrm{~Hz}$ vibration, or placebo. No significant changes in thermal sensitivity were observed during and after conditioning stimulation in any of the test groups, although $24 / 40(60 \%)$ of the patients reported reduction of their clinical pain intensity. The results indicate that (a) thermal sensitivity is not influenced by the presence of clinical pain, (b) the effects of stimulation on thermal sensitivity (thresholds) and clinical pain are not closely related, (c) central inhibitory effects of TENS and vibration are crucial for their pain relieving capacity.

Several studies have been performed to elucidate the pain reducing effect of transcutaneous electrical nerve stimulation (TENS) in experimentally induced pain in healthy volunteers as well as in pain patients. Few studies have actually compared the effect of afferent stimulation on experimental and clinical pain in the same patient (cf. ref. 1). Such a comparison seems important since studies on experimental pain in healthy subjects and pain patients have yielded some conflicing results (cf. ref. 1).

In the present study we have compared the effect of different types of stimulation, that is, low and high frequency TENS as well as vibration and placebo, on both experimental and clinical pain. Earlier reports on TENS $^{2}$ and dorsal column stimulation (DCS) ${ }^{3}$ have dealt with long-standing chronic pain. We have concentrated our efforts on patients with acute pain of short duration.

First, we have observed to what extent on-going clinical pain influences temperature and pain perception, intra- and extrasegmentally with regards to the origin of the pain. This might be of interest regarding the development of sensory abnormalities due to

Address for reprint requests: Dr Anders Ekblom, Department of Physiology II, Karolinska Institutet, S-104 01 Stockholm, Sweden.

Received 22 April 1986 and in revised form 24 November 1986. Accepted 28 November 1986 interaction between activity in different sets of nerve fibres subserving various sensory modalities. ${ }^{4}$

Secondly, we have tried to find out if there is $\alpha$ correlation between the effect of afferent stimulation on experimental versus clinical pain. If such a coro relation exists, it could be used in a predictive manneto in pain treatment with methods such as TENS.

\section{Material and methods}

The study was carried out on 40 patients, 23 males and 17 females aged $20-58$ years and on six pain-free subjects, three males and three females, aged 29-47 years.

\section{Clinical pain}

Patients were admitted to an emergency clinic for dental and oral surgery due to acute pain from teeth and/or surrounding tissues. The pain was due to pulpal inflammation apical periodontitis, pericoronitis or postoperative pain following operative removal of an impacted tooth. In all cases pain was ipsilaterally perceived corresponding to the trigeminal branch innervating the area of the inflammatory lesion. The affected tissue was innervated either by the maxillar (13 cases) or the mandibular ( 27 cases) division.

The patients had suffered pain for 1-4 days. No patient had taken any analgesics within at least 10 hours before experimental procedures. All patients reported constant pain, that is not varying more than $\pm 10 \%$ of its intensity over the hour.

All patients were examined, told their diagnosis and asked if they would take part in the experiments. If they agreed to participate, they were informed about the experimental pro- 
cedures in general, and that they could stop the procedures at any moment they desired. They were also told that they would get conventional dental treatment following the test session.

The subjects were informed that they might experience pain alleviation, no change or pain aggravation during stimulation. Care was taken to avoid suggestion.

Subjects were assigned to one of five groups: (1) vibration $100 \mathrm{~Hz}$, eight patients, (2) placebo vibration, five patients, (3) $100 \mathrm{~Hz}$ TENS, 11 patients, (4) $2 \mathrm{~Hz}$ TENS, 11 patients, and (5) placebo TENS, 5 patients.

All patients rated their pain intensity initially using a graded five level verbal scale: light, light-moderate, moderate, moderate-severe and severe pain; and a visual analogue scale (VAS) both initially and following stimulation. The VAS consisted of a $10 \mathrm{~cm}$ horizontal line on a card. The words "no pain" and "worst pain ever" were placed on the left and right extreme ends of the line, respectively. Patients were instructed to mark the line at a point representing their pain.

During the experimental session each patient continuously rated his/her pain intensity using a graphic rating scale (GRS) consisting of a lever attached to a potentiometer controlling the position of a pen on a chart-recorder out of sight of the patient. The patients were instructed to move the lever from zero position (indicating pain intensity before starting stimulation) to one side when pain was reduced (endpoint $=10$, meaning no pain) and to the opposite side if pain increased in intensity (endpoint $=10$, meaning worst pain ever). Evaluation of the obtained pain reduction during stimulation was performed using the GRS for continuous registration. The values obtained with the VAS and GRS were consistent.

\section{Thermal sensibility}

All patients, regardless of the kind of conditioning stimulation used, were tested with respect to warm-cold, and thermal heat pain perception.

Thermal stimulation was delivered to the skin using a feed-back controlled thermode, consisting of four Peltier elements with an interposed thermocouple, stimulus surface $10 \times 10 \mathrm{~mm}$. The output current from the thermocouple measuring the temperature of the skin at the stimulus/probe interface was amplified and fed to the control unit for the Peltier elements. The side of the Peltier elements not facing the skin was cooled/heated by circulating water through a small chamber attached to this side with thermally conducting epoxi. The stimulating surface of the Peltier elements could be heated or cooled depending on the direction of current through the elements, with a temperature change which was linear over the temperature range $20-60^{\circ} \mathrm{C}$. Measurements were made with a temperature change of $0.8^{\circ} \mathrm{C} / \mathrm{s}$. All measurements were started from an adapting temperature of $34-35^{\circ} \mathrm{C}$, the same in all tests for each individual. Measurements were made from recordings on a chartrecorder of the output from the thermocouple.

Warm-cold perception Warm and cold thresholds were measured using the technique described by Fruhstorfer et $a l .{ }^{5}$ The thermode was applied to the skin and the patient was instructed to press the button of a hand-held switch at the first sensation of warmth: this reversed the current to the Peltier elements shifting the thermode temperature in the cooling direction. The patient was also instructed to press the button of the switch at the first sensation of cold thereby shifting the thermode temperature in the warming direction. This procedure was repeated several times until stable values were reached. Determination of warm and cold thresholds was made from the last three to five measurements and the means were taken.

Pain threshold The probe temperature was continuously increased until the subject reported the stimulus as painful, at which instant the current to the Peltier elements was reversed by the subject, using the hand-held switch, returning the thermode to start temperature. Pain thresholds were measured twice and the mean was taken.

\section{Conditioning stimulation}

Mechanical vibratory stimulation The vibrator (Bruel \& Kjaer, 4806) was driven by sinuoisodal pulses at $100 \mathrm{~Hz}$. Stimulation amplitude was $400-800 \mu \mathrm{m}$. The disc-shaped probe, diameter $3 \mathrm{~cm}$, was applied at right angles to the skin in an attempt to exert pressure on the underlying bone.

TENS $(2 \mathrm{~Hz}$ and $100 \mathrm{~Hz})$ The stimulator produced monopolar square wave pulses of $0.2 \mathrm{~ms}$ duration at $100 \mathrm{~Hz}$ or a $71 \mathrm{~Hz}$ pulse train (duration $84 \mathrm{~ms}$ ) at $2 \mathrm{~Hz}^{6} \mathrm{~A}$ pair of conducting rubber electrodes, each measuring $3 \times 3 \mathrm{~cm}$, was positioned on the skin overlying the painful area. The most distally placed electrode was always connected to the anode and the proximal electrode to the cathode. With $100 \mathrm{~Hz}$ TENS the stimulus intensity was set to give a tingling sensation and with $2 \mathrm{~Hz}$ TENS prominent muscular contractions. The TENS stimulation was never reported as painful.

Placebo (vibration and TENS) Placebo stimulation was accomplished by applying the vibrator probe or TENS electrodes in contact with the skin of the painful area as for active stimulation but without transmitting any actual vibratory or electrical stimulation. The general procedure was the same as for the patients receiving vibration or TENS except that the patients were informed that some people might not experience the stimulation.

\section{General experimental procedure}

Before starting conditioning stimulation the thresholds for warmth, cold and pain were assessed: (A) on the skin within the painful area distal to the vibrator probe and the TENS electrodes respectively (the distance between thermode and the distal electrode/probe varied between 2-4 cm); (B) on a corresponding contralateral area; (C) on the dorsal aspect of the hand, ipsilateral to the painful side, in order to study extrasegmental effects.

Warm-cold thresholds and heat pain threshold measurements were made at separate spots, and the interval between successive stimulations at a certain spot was in general 15 mın in order to avoid skin sensitisation or suppression. ${ }^{7}$ The same areas were used for each test before, during, and after conditioning stimulation. Measurements were always made in the order of warm-cold and pain threshold. Next, conditioning stimulation (CS) was started. Fifteen minutes later, during CS, the same measurements were repeated. After a total time of 30 min CS was terminated. Fifteen min later measurements were repeated. 


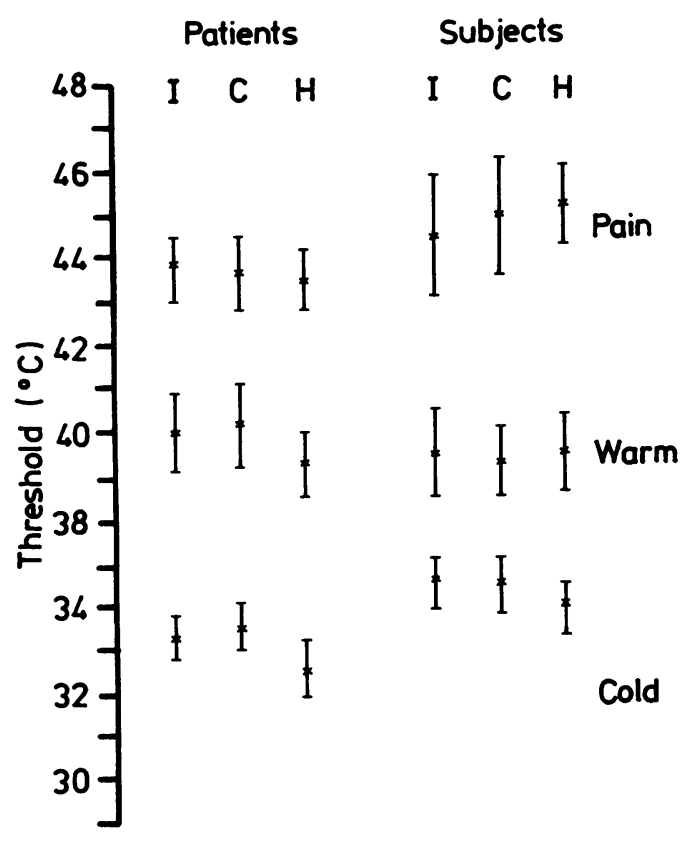

Fig 1 Average cool, warm and heat pain thresholds for all patients (left side diagrams) and pain-free subjects (right side diagrams) in different skin areas. Vertical bars represent $95 \%$ confidence limits. $I=$ skin area innervated by trigeminal branch carrying the pain (patients) or corresponding skin area in subjects. $C=$ contralaterally located skin area, corresponding to painful area. $H=$ dorsal aspect of the hand, ipsilateral to the painful side.

\section{Control group}

In order to compare the influence of CS on perception of experimental stimulation in patients having clinical pain with that of pain-free subjects, six healthy normal volunteers were tested with the same types of CS and experimental parameters as those described above.

\section{Statistical analysis}

Analysis of temperature thresholds before, during, and after stimulation was made with a general analysis of variance, ANOVA. ${ }^{8}$ The ANOVA was made with conditioning stimulation, skin areas, and time as fixed factors. To approximately fulfil the assumption for the ANOVA, threshold values were transformed to logarithms before the analysis. Comparison of the number of patients reporting pain reduction using the different methods were made using the chisquare test. The significance level $p<0.05$ was considered significant in the statistical analysis.

\section{Results}

Temperature and pain perception Mean temperature

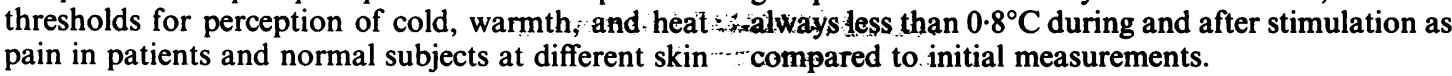




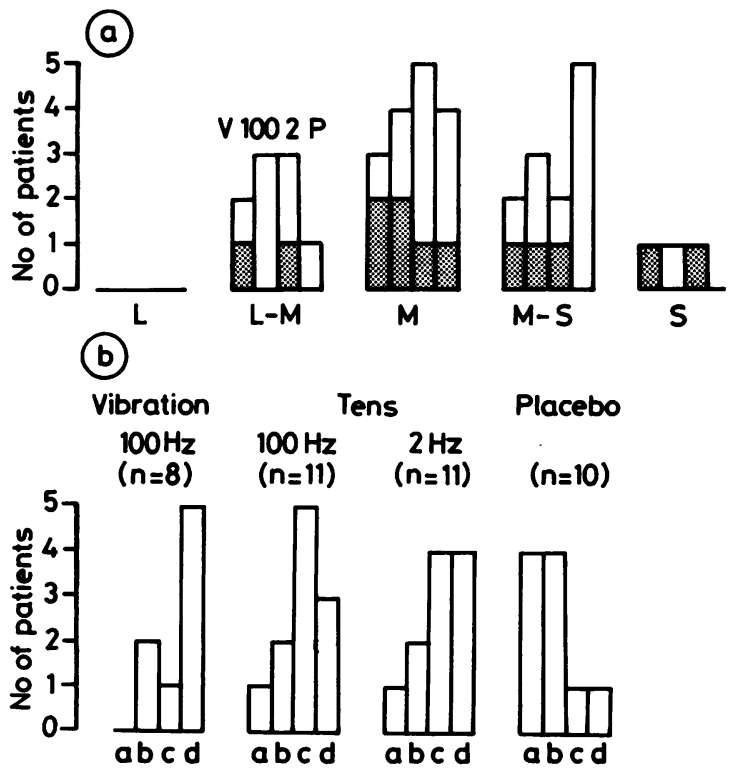

Fig 3 (A) Number of patients with different degrees of pain ( $L=$ light pain, $M=$ moderate pain, $S=$ severe pain) who received $100 \mathrm{~Hz}$ vibration (V), $100 \mathrm{~Hz} T E N S(100), 2 \mathrm{~Hz}$ TENS (2), placebo-TENS or vibration (P). Patients who experienced pain reduction exceeding $50 \%$ represented by hatched areas. (B) Effects of conditioning stimulation on subjective pain intensity (a) pain increase; (b) no change in pain intensity; (c) pain reduction less than $50 \%$; (d) pain reduction exceeding $50 \%$.

No correlation was found between a change in clinical pain intensity and a change in cold, warm and heat pain thresholds during or after conditioning stimulation.

Clinical pain The distribution of patients having different pain intensities prior to stimulation, as rated on the verbal scale, is given in fig $3 \mathrm{~A}$ showing a roughly even distribution.

The effect of TENS, vibration, and placebo on subjective pain intensity is seen in fig 3B. Of all patients tested, three reported complete pain relief (one patient receiving vibration and two patients receiving $100 \mathrm{~Hz}$ TENS). No type of active stimulation was superior to the others concerning number of patients reporting pain reduction. Placebo was significantly less effective than active stimulation.

\section{Discussion}

Thermal sensitivity before conditioning stimulation The cold and heat pain thresholds both in patients and in pain-free subjects were in the same range as reported by others, ${ }^{5910}$ although warm thresholds tended to be somewhat higher than those previously described. This difference in findings might be due to the difference in stimulating area of the thermode, in this study $10 \times 10 \mathrm{~mm}$ as compared with $25 \times 25 \mathrm{~mm}$ used by others, ${ }^{510} 11$ since thermal thresholds depend on spatial summation. ${ }^{12}$

The findings that thresholds did not differ in various skin areas in patients or subjects, or between patients and subjects indicate that the ongoing acute pain did not influence thermal sensitivity significantly. Lindblom and Meyerson ${ }^{3}$ did not find any relationship between chronic pain and perception of mechanically induced cutaneous pain, except in one patient with an abnormally low pain threshold in a hyperaesthetic area. Ischaemic pain has, however, been reported to elevate dental pain thresholds and decrease (non significant) thermal sensitivity in healthy subjects. ${ }^{13}$

Thermal sensitivity during and after conditioning stimulation The present finding that TENS and vibration did not change thermal sensitivity, even in the painful and stimulated area, is in agreement with reports on the effect of $\mathrm{DCS}^{3}$ and intracerebral stimulation ${ }^{1415}$ on experimental pain in chronic pain patients. A comparison between those former and the present results indicates that the duration of pain does not seem to be a crucial factor in determining the degree of interaction between pain and thermal sensitivity or the influence of TENS and vibration on activity in those systems. It seems that there is a rather secure transmission of the thermally induced activity from the skin. ${ }^{16}$ However, in dysaesthetic skin areas a parallel increase has been reported between mechanical pain thresholds and reduction of chronic pain. ${ }^{3}$

The finding that conditioning stimulation did not affect skin heat pain thresholds but did reduce clinical pain is interesting since in both areas activity in smalldiameter nerve fibres transmitted to similar second order neurons is thought to be responsible for the perceived sensations (cf. ref. 17). Possible explanations for this discrepancy could be the following.

First, the peripheral site of origin might be of importance. The temperature stimulation was given to the skin whereas the clinical pain originated more deeply and was probably mediated by activity in deep somatic afferents. The present results could reflect a difference in susceptibility to central modulation induced by TENS and vibration on input from small diameter cutaneous and deep somatic afferents, as proposed by Woolf. ${ }^{10}$ Thus, TENS and vibration have been shown to increase the tooth pain threshold, ${ }^{18-20}$ and high-frequency TENS to reduce ischaemic pain ${ }^{10}$ in normal subjects but negative findings have been reported using cutaneously applied mechanical and thermal stimuli. ${ }^{1021}$

Secondly, a difference in impulse pattern in the fibres activated by the thermal stimulation as com- 
pared with the one set up by the pathological process might also be of importance. The thermal stimulation with a rather rapid change in temperature would be expected to give a more synchronous activation of afferent nerve fibres than that seen during the constant clinical pain. In a previous study ${ }^{22}$ two types of painful stimulation, probably creating different afferent temporal and spatial input patterns, were differently susceptible to TENS and vibration, that is, acute oro-facial pain due to pathology but not the pain induced by operative procedures was reduced by TENS and vibration.

It has been suggested that at least part of the painreducing effect induced by TENS is caused by a peripheral blockade or fatigue of pain transmitting fibres $^{23-25}$ although this has been rejected by Janko and Trontelj. ${ }^{26}$ The present finding that stimulation had no effect on the thermal thresholds but diminished clinical pain would argue against such peripheral effects of TENS considering local anatomy. No reports exist demonstrating nociceptive afferents with peripheral divergens innervating both the tooth pulp, the surrounding tissues as well as cutaneous areas of the face. Such afferents would be a prerequisite to induce direct peripheral effects by TENS.

The present data also argue against distraction by the afferent stimulation as an alternative explanation for its pain reducing effect. The paraesthesia induced by the conditioning stimulation disappeared shortly after termination of stimulation, whereas pain reduction persisted for a period of $15 \mathrm{~min}$ to 5 hours.

The authors thank Dr Jan Lännergren for constructive criticism on the manuscript and Professor Allen Counter for the revision of the English text. Mrs Monica Thunberg-Eriksson, Ms Maud Hoffstedt and Ms Ulla Lindgren are thanked for skilful secretarial assistance.

\section{References}

1 Meyerson B. Electrostimulation procedures: effects, presumed rationale, and possible mechanisms. In: Bonica $\mathrm{JJ}$, Lindblom U, Iggo A, eds. Advances in Pain Research and Therapy. New York: Raven Press, 1983:495-534.

2 Callaghan M, Sternbach RA, Nyquist JK, Timmermans G. Changes in somatic sensibility during transcutaneous electrical analgesia. Pain 1978;5:115-28.

3 Lindblom U, Meyerson B. Influence on touch, vibration and cutaneous pain of dorsal column stimulation in man. Pain 1975; 1:257-70.

4 Lindblom U. Assessment of abnormal evoked pain in neurological pain patients and its relation to spontaneous pain: a descriptive and conceptual model with some analytical results. In: Fields HL, Dubner R, Cervero F, eds. Advances in Pain
Research and Therapy. New York: Raven Press, 1985:409-23.

5 Fruhstorfer H, Lindblom U, Schmidt WG. Method for quantitative estimation of thermal thresholds in patients. $J$ Neurol Neurosurg Psychiatry 1976;39:1071-5.

6 Eriksson MBE, Sjölund BH. Acupuncture-like electroanalgesia in TNS-resistant chronic pain. In: Zotterman Y, ed. Sensory Functions of the Skin in Primates, with Special Reference to Man. Oxford: Pergamon Press, 1976:575-81.

7 Price DD, Hu JW, Dubner R, Gracely RH. Peripheral suppression of first pain and central summation of second pain evoked by noxious heat pulses. Pain 1977;3:57-68.

8 Snedocor GW, Cochran WG. Statistical Methods. Iowa State University Press, 1980.

9 Pertovaara A, Kojo I. Influence of the rate of temperature change on thermal thresholds in man. Exp Neurol 1985;87:439-45.

10 Woolf CJ. Transcutaneous electrical nerve stimulation and the reaction to experimental pain in human subjects. Pain 1979;7:115-27.

11 Fagius J, Wahren LK. Variability of sensory threshold determination in clinical use. J Neurol Sci 1981;51:11-27.

12 Kenshalo DR. Psychophysical studies of temperature sensibility. In: Neff WD, ed. Contributions to Sensory Physiology. New York: Academic Press, 1970;4:17-24.

13 Pertovaara A, Kemppainen P, Johansson G, Karonen S-L. Ischaemic pain non-segmentally produces a predominan reduction of pain and thermal sensitivity in man: a selective role for endogenous opioids. Brain Res 1982;251:83-92.

14 Gybels JM. Electrical stimulation of the central gray for pain relief in humans: a critical review. In: Bonica JJ, Liebeskind JC, Albe-Fessard DG, eds. Advances in Pain Research and Therapy. New York: Raven Press, 1979:499-509.

15 Hosobuchi Y, Adams JE, Linchitz R. Pain relief by electricab stimulation of the central gray matter in humans and its rever sal by naloxone. Science 1977;197:183-6.

16 Sumino R, Dubner R. Response characteristics of specific thermoreceptive afferents innervating monkey facial skin ando their relationship to human thermal sensitivity. Brain Res Rev 1981;3:105-22.

17 Willis WD. The pain system: the neural basis of nociceptive trans mission in the mammalian nervous system. In: Gildenber PhL, ed. Pain and Headache. Basel: Karger, 1985;8:22-54

18 Andersson SA, Holmgren E. On acupuncture analgesia and the mechanism of pain. Am J Chin Med 1975;3:311-34.

19 Kemppainen P. Modification of human dental-pain thresholds by conditioning vibrotactile stimulation at high-frequency. Arch Oral Biol 1983;10:953-62.

20 Pertovaara A, Kemppainen P, Johansson G, Karonen S-L. Dental analgesia produced by non-painful low-frequency stimulation is not influenced by stress or reversed by naloxone. Pain 1982;13:379-84.

21 Nathan PW, Rudge P. Testing the gate-control theory of pain in man. J Neurol Neurosurg Psychiatry 1974;37:1366-72.

22 Hansson P, Ekblom A. Afferent stimulation induced pain relief in acute oro-facial pain and its failure to induce sufficient pain reduction in dental and oral surgery. Pain 1984;20:273-8.

23 Campbell JN, Taub A. Local analgesia from percutaneous electrical stimulation. Arch Neurol 1973;28:347-50.

24 Ignelzi RJ, Nyquist JK. Direct effect of electrical stimulation on peripheral nerve evoked activity: implications in pain relief J Neurosurg 1976;45:159-66.

25 Pertovaara A. Experimental pain and transcutaneous electrica nerve stimulation at high-frequency. Appl Neurophysiol 1980 43:290-7.

26 Janko M, Trontelj JV. Transcutaneous electrical nerve stimulation: a microneurographic and perceptual study. Pain 1980 9:219-30. 